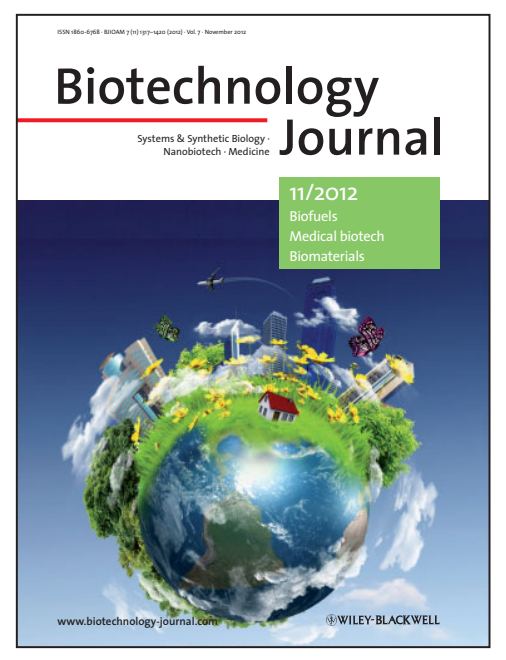

Cover illustration

This "regular" issue of Biotechnology Journal gathers the state-of-the-art in biotechnology, with articles on bioenergy, biofuels, medical biotechnology, biomaterials, etc.

Image: (c) Sergej Khackimullin - Fotolia.com.

Biotechnology Journal - list of articles published in the November 2012 issue.

Perspective

Orgenic plants: Gene-manipulated plants compatible with organic farming

Gerhart U. Ryffel

http://dx.doi.org/10.1002/biot.201200225

Rapid Communication

Efficient reversal of phiC31 integrase recombination in mammalian cells

Alfonso P. Farruggio, Christopher L. Chavez,

Carlos L. Mikell and Michele P. Calos

http://dx.doi.org/10.1002/biot.201200283

Research Article

Exploring the combinatorial genomic space

in Escherichia coli for ethanol tolerance

Sergios A. Nicolaou, Stefan M. Gaida

and Eleftherios T. Papoutsakis

http://dx.doi.org/10.1002/biot.201200227

Research Article

Production of human cytochrome P450 2D6 drug metabolites with recombinant microbes - a comparative study Martina Geier, Andreas Braun, Anita Emmerstorfer, Harald Pichler and Anton Glieder

http://dx.doi.org/10.1002/biot.201200187

Research Article

Recombinantly produced cellobiose dehydrogenase

from Corynascus thermophilus for glucose biosensors and biofuel cells

Wolfgang Harreither, Alfons K. G. Felice, Regina Paukner, Lo Gorton, Roland Ludwig and Christoph Sygmund http://dx.doi.org/10.1002/biot.201200049

Research Article

Molecular dynamics simulation of the induced-fit binding process of DNA aptamer and L-argininamide

Po-Hsun Lin, Ching-Wei Tsai, Josephine W. Wu,

Ruoh-Chyu Ruaan and Wen-Yih Chen

http://dx.doi.org/10.1002/biot.201200003
Research Article

Wound-healing evaluation of entrapped active agents into protein microspheres over cellulosic gauzes Raquel Silva,Helena Ferreira, Teresa Matamá, Andreia C. Gomes and Artur Cavaco-Paulo http://dx.doi.org/10.1002/biot.201200142

Research Article

Biomimetic soluble collagen purified from bones Ana Marina Ferreira, Piergiorgio Gentile, Susanna Sartori, Cristina Pagliano, Chiara Cabrele, Valeria Chiono and Gianluca Ciardelli

http://dx.doi.org/10.1002/biot.201200184

Research Article

Gene network analysis leads to functional validation of pathways linked to cancer cell growth and survival Emmanuelle Berger, Nathalie Vega, Hubert Vidal and Alain Geloën

http://dx.doi.org/10.1002/biot.201200188

Technical Report

Albumin 3'untranslated region facilitates increased recombinant protein production from Chinese hamster ovary cells

Mark J. Pearson, Siavash Khazaipoul, Asta Optun, Ian F. Pryme, Beate Stern and John E. Hesketh http://dx.doi.org/10.1002/biot.201200044

Technical Report

Photosynthetic efficiency and rate of $\mathrm{CO}_{2}$ assimilation by Arthrospira (Spirulina) platensis continuously cultivated in a tubular photobioreactor

Marcelo Chuei Matsudo, Raquel Pedrosa Bezerra, Sunao Sato, Attilio Converti and João Carlos Monteiro de Carvalho

http://dx.doi.org/10.1002/biot.201200177 


\title{
Wound-healing evaluation of entrapped active agents into protein microspheres over cellulosic gauzes
}

\author{
Raquel Silva ${ }^{1}$,Helena Ferreira ${ }^{7}$, Teresa Matamá ${ }^{1}$, Andreia C. Gomes ${ }^{2}$ and Artur Cavaco-Paulo \\ ${ }^{1}$ University of Minho, Department of Textile Engineering, Campus de Azurém, Guimarães, Portugal \\ ${ }^{2}$ Molecular and Environmental Biology Centre (CBMA), Department of Biology, University of Minho, Campus of Gualtar, Braga, \\ Portugal
}

\begin{abstract}
The use of active ingredients in wound management have evolved alongside the pharmaceutical agents and dressings used to deliver them. However, the development of gauzes, dressings with specific properties, still remains a challenge for several medical applications. A new methodology for the controlled release of active components for the healing of burn wounds is proposed herein. Cotton and non-woven bandages have been cationised to promote the attachment of protein microspheres. The active agents, piroxicam and vegetable oil, were entrapped into the microspheres using ultrasound energy. Active agents were released from the microspheres by a change in $\mathrm{pH}$. Wound healing was assessed through the use of standardised burn wounds induced by a cautery in human full-thickness skin equivalents (EpidermFT). The best re-epithelialisation and fastest wound closure was observed in wounds treated with proteinaceous microspheres attached to gauzes, after six days of healing, in comparison with commercial collagen dressing and other controls. Furthermore, the ability of these materials to reduce the inflammation process, together with healing improvement, makes these biomaterials suitable for wound-dressing applications.
\end{abstract}

$\begin{array}{ll}\text { Received } & \text { 21 MAR } 2012 \\ \text { Revised } & \text { 23 MAY } 2012 \\ \text { Accepted } & \text { 13 MAR } 2012 \\ \text { Accepted } & \\ \text { article online } & 10 \text { JUL } 2012\end{array}$

Keywords: Gauzes · Inflammation · Non-steroidal anti-inflammatory drugs · Protein microspheres · Wound healing

\section{Introduction}

The use of textiles in medicine has a long tradition. An important field of application is in wound care and prevention of chronic wounds [1]. Among the long list of textile materials, bandages and wound dressings gained great popularity [2]. The exploitation of textile materials was supported by availability, prices and re-usability [3]. Along with the technological development of functional textiles, their use in wound healing and prevention of chronic wounds has reached a new quality of interactiv-

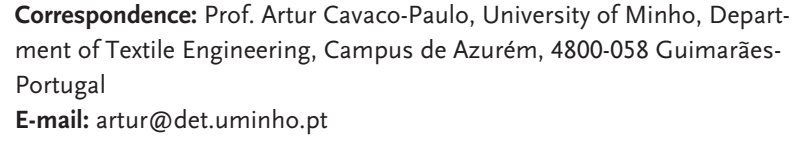
ment of Textile Engineering, Campus de Azurém, 4800-058 GuimarãesPortugal

E-mail: artur@det.uminho.pt

Abbreviations: BSA, bovine serum albumin; COX, cyclo-oxygenase; NSAID, non-steroidal anti-inflammatory drug; PBS, phosphate-buffered saline; PDDA, poly(diallyldimethylammonium chloride; SD, Standard deviation; SEM, scanning electron microscopy; ity between biological tissues and textiles [2]. These fibres were shown to favour wound occlusion, exudate transport and drug release with much reduced distress to the patient [1]. Despite the fact that natural textiles fulfilled primary quality approaches, such as biocompatibility, flexibility and strength, there is an increasing need for specified functions [4]. In particular, the controlled release of bioactive molecules to counteract the progression of infection and inflammation, such as in chronic wound management, was considered a step forward in this research area [5]. The local delivery of drugs by topical administration or a delivery device may enable the maintenance of a high local drug concentration for an extended period without reaching systemic toxicity. This is an important feature because the use of anti-inflammatory drugs, such piroxicam, which is a non-steroidal antiinflammatory drug (NSAID), is very useful to counteract local inflammation, although it can be absorbed through blood vessels and could result in severe side effects for both the gastrointestinal tract and kidneys[6]. Thus, the topical administration of NSAIDs can control these se- 
vere side effects. Besides this advantage over conventional oral and intravenous dosage forms, topical drug delivery offers many other benefits, such as first pass metabolism avoidance, pain minimisation and patient compliance [7]

Controlled delivery dressings can provide an excellent means of delivering drugs to wound sites in a consistent and sustained manner over long periods of time without the need for frequent dressing changes[8].Therefore, there is a need to develop new dressings that are useful in the treatment of local infections, where increasing the local concentrations of anti-inflammatory drugs may be beneficial, while avoiding high systemic doses; thus reducing patient exposure to an excess of drug beyond that required at the wound site $[9,10]$. Furthermore, a dressing that delivers an active substance to a wound site in a controlled fashion for a sustained period of about a week could help solve or minimise issues associated with noncompliance patients.

Several biodegradable devices from both natural and synthetic polymers have been produced by different processes in recent years for use as drug carriers. Biodegradable polymers can release larger quantities of pharmaceutical agents and their degradation properties can be tailored for a specific application [11]. Natural polymers, such as proteins, are attractive, since they exhibit superior biocompatibility and biodegradability [12]. Recently, proteinaceous microspheres were developed that incorporated different oils to control the high levels of elastase produced in chronic wounds [13]. Due to its involvement in such process, there is extensive literature exploring different types of inhibitors or inhibitor formulations that may restore the normal levels of this enzyme [14]. To the best of our knowledge, anti-inflammatory drugs have been attached onto cotton fibres [15], but never in combination with oils.

In the present work, piroxicam, sonochemically entrapped to bovine serum albumin (BSA) microspheres, was attached to cotton and non-woven gauzes to produce a functionalised biomaterial with the ability to deliver the pharmaceutical agent in a controlled manner. Moreover, the wound-healing ability of these materials was tested on 3D human skin equivalents.

\section{Materials and methods}

\subsection{Materials}

Piroxicam was acquired from Sigma (Spain), along withpolyvinyl alcohol (PVA; MW = 30 000-70 000 Da, 87-90\% hydrolysed) and BSA. The centricon tubes (molecularweight cutoff of $100 \mathrm{kDa}$, Amicon) were purchased from Amicon.

The sterilised gauzes fibres were commercially available from a local pharmacy (100\% cotton gauze and non- woven gauze composed of $67 \%$ viscose and 33\% polyester).

Human full-thickness skin equivalents (EpidermFT) were supplied by MakTek Corporation (USA). All other reagents were of analytical grade and purchased from Sigma (Spain).

\subsection{Equipment}

The experimental setup used was composed of a probetype ultrasound source (20kHz Sonics \& Materials Vibracell CV 33) fitted with a $3 \mathrm{~mm}$ diameter titanium micro-tip. Power delivery was controlled as percentage amplitude. The reaction vessel was an open glass cell (diameter $19 \mathrm{~mm}$, height $75 \mathrm{~mm}$ ), which contained $16 \mathrm{~mL}$ of sample solution. The sonochemical reactor temperature was controlled by a thermostated water bath with a freezer exchanger placed within a thermo jacket cell; this gave a steady operating temperature of $(10 \pm 1)^{\circ} \mathrm{C}$. The temperature was monitored throughout using $\mathrm{K}$-type thermocouples.

\subsection{Sonochemical preparation of proteinaceous microspheres}

Microspheres, with NSAID, were synthesised by an adaptation of the Suslick method [16].Piroxicam $(3000 \mu \mathrm{M})$ was added to protein solution $\left(5 \mathrm{~g} \mathrm{~L}^{-1}\right)$ and layered with vegetable oil. The very low solubility of piroxicam required the use of dimethylsulfoxide/water solutions (1:99 v/v). Finally, the surfactant, used as stabiliser, was dissolved in the aqueous phase. The bottom of the high-intensity ultrasonic horn was positioned at the aqueous/organic interface, employing an amplitude of $40 \%$ with a temperature of $(10 \pm 1)^{\circ} \mathrm{C}$ and with a total treatment time of $3 \mathrm{~min}$. The separation of phases was accomplished in a few minutes, due to the lower density of microspheres, relative to the density of water. However, to obtain complete separation of the proteinaceous microspheres from the mother solution, the flasks were placed in a refrigerator $\left(4^{\circ} \mathrm{C}\right)$ for $24 \mathrm{~h}$. Microspheres were later collected by centrifugation (2000 g, $30 \mathrm{~min}$ ) using the centricon tubes.

\subsection{Microsphere characterisation parameters}

\subsubsection{Determination of microsphere yield}

The efficiency of microsphere formation was determined by the Lowry method [20], using BSA as a standard. This method was based on the quantification of protein in the supernatant. The efficiency of microsphere formation was calculated from Eq. (1):

$$
\begin{aligned}
& \text { Microsphere formation }(\%) \\
& =\frac{[p]_{\text {total }}-[p]_{\text {supernatant }}}{[p]_{\text {total }}} \times 100
\end{aligned}
$$


in which $[p]_{\text {total }}$ and $[p]_{\text {supernatant }}$ are the initial and final protein concentrations in the aqueous solution, respectively. This process indicates the percentage of protein that formed microspheres in relation to the total amount used during preparation.

Measurements were recorded in triplicate and the results were expressed as mean value \pm standard deviation (SD).

\subsubsection{Determination of size and zeta-potential}

The size distributions and zeta-potentials of the microspheres were determined at $(25.0 \pm 0.1)^{\circ} \mathrm{C}$ using a Malvern zetasizer NS (Malvern Instruments) by photon correlation spectroscopy (PCS) and electrophoretic laser Doppler anemometry, respectively. The proteinaceous microspheres were diluted in phosphate-buffered saline (PBS) 1:100. The results were expressed as mean value \pm SD.

\subsubsection{Entrapment efficiency of piroxicam}

The entrapment efficiency of piroxicam in proteinaceous microspheres was measured by UV spectrophotometry at $353 \mathrm{~nm}$. The separation of free piroxicam was achieved by centrifugation of samples at $1500 \mathrm{~g}$ for $30 \mathrm{~min}$, using centricon tubes. The entrapment efficiency of piroxicam was obtained from Eq. (2):

$$
\begin{aligned}
& \text { Entrapment }(\%) \\
& =\frac{[\text { piroxicam }]_{\text {initial }}-[\text { piroxicam }]_{\text {final }}}{[\text { piroxicam }]_{\text {final }}} \times 100
\end{aligned}
$$

in which [piroxicam $]_{\text {initial }}$ is the initial concentration used and [piroxicam] $]_{\text {final }}$ is the final concentration in the supernatant.

\subsection{Chemical activation of gauzes}

\subsubsection{Cationisation of cotton and non-woven gauzes}

The gauzes were cationised using the exhaust method as described in [17]. Cationisation was performed in sealed, stainless-steel pots with $120 \mathrm{~cm}^{3}$ capacity in a laboratoryscale dyeing machine (AHIBA Spectradye, from Datacolor International) with IR temperature control.

The treatment was carried out using a material/liquor ratio of $1: 20,10 \%$ (on weight of the fabric) of poly(diallyldimethylammonium chloride) (PDDA) and $5.0 \mathrm{~g} \mathrm{~L}^{-1}$ of sodium hydroxide over $60 \mathrm{~min}$ at $50^{\circ} \mathrm{C}$. Then the gauzes were washed thoroughly with distilled water.

\subsubsection{Determination of the cationisation efficiency on the gauze surface}

Cationisation at the surface of gauzes (cotton and nonwoven) was analysed by staining of treated samples and controls (cotton and non-woven gauzes without cationisation treatment) with Comassie brilliant blue G250 (Acid Blue 90; C.I. 42655). The staining was executed in an AHIBA machine using $1 \mathrm{~g} \mathrm{~L}^{-1}$ of Comassie dye, at $60^{\circ} \mathrm{C}$ for
60 min. After staining, the samples were washed with distilled water until no more dye could be detected in the washing solution. Colour measurements were determined using a spectrophotometer (illuminant $D_{65}$ at 600nm; Spectraflash, from Datacolor International) coupled to a computer. The colour strength was evaluated as $K / S$ (Eq. 3) at the maximum absorption wavelength $(600 \mathrm{~nm})$. The ratio between the absorption $(K)$ and scattering $(S)$ coefficients is related to reflectance data by applying Kubelka-Munk's law at each wavelength, and it is proportional to dye concentration $[18,19]$. $R$ is the light reflection. For all types of gauze samples, experiments were performed in triplicate.

$\frac{K}{S}=\frac{1-R^{2}}{2 R}$

\subsection{Attachment of proteinaceous microspheres, containing piroxicam, onto gauze bandages}

To attach proteinaceous microspheres containing piroxicam to the gauzes $(0.1 \mathrm{~g})$, two sets of experiments were applied, namely, silent (incubation) and sonochemical methods. The attachment of microspheres by incubation was carried out in two different steps. Firstly, the microspheres were produced and submitted to an extensive physico-chemical characterisation. They were later incubated with the gauzes in a water shaking bath at room temperature $\left(25^{\circ} \mathrm{C}\right)$ for $24 \mathrm{~h}$ under constant shaking (50 rpm). Several controls were run simultaneously: a control test with non-cationised gauzes (cotton and non-woven) and a control test with cationised gauzes (cotton and non-woven) without microspheres, incubated with PBS, $0.01 \mathrm{M}(\mathrm{pH}=7.4)$. After incubation, the samples were removed and washed with distilled water.

Secondly, attachment of proteinaceous microspheres by the sonochemical method was performed in a one-step reaction. In this case, the gauzes were added to the biphasic system, consisting of protein solution and vegetable oil, by applying the same conditions previously described. The control samples were gauzes (cotton and non-woven, cationised and non-cationised) in PBS submitted to ultrasound treatment under the same conditions. The remaining solution was centrifuged, using centricon tubes, and the protein concentration in the supernatant and the amount of piroxicam entrapped was determined. Finally, the sample gauzes were collected and washed with distilled water. All measurements were performed in triplicate.

\subsection{Evaluation of proteinaceous microspheres attached to gauzes based on the ninhydrin reaction}

The presence of protein microspheres in the gauze samples was evaluated using ninhydrin (2,2-dihydroxyin- 
dane-1,3-dione) reagent. Ninhydrin is most commonly used to detect terminal amines or lysine residues in peptides and proteins [18]. When reacting with these free amines, a deep blue or purple colour, known as Ruhemann's purple, is produced.

For this assay, the gauzes were dried at $60^{\circ} \mathrm{C}$ and then incubated with ninhydrin solution $(0.5 \%)$, at $60^{\circ} \mathrm{C}$, for $30 \mathrm{~min}$. This test was performed on the samples after proteinaceous microsphere attachment by incubation and ultrasound treatment, and on control gauzes (cotton and non-woven samples gauzes, cationised and noncationised without microspheres).

\subsection{Scanning electron microscopy (SEM) analysis}

SEM images were obtained with a scanning electronic microscope (NOVA Nano SEM 200, FEI) with detection of backscattered and secondary electrons. The samples were previously gold coated in vacuum.

\subsection{Quantification of piroxicam released from proteinaceous microspheres attached to gauze bandages}

The release of piroxicam by the microspheres attached to gauzes was examined by incubation of the textile samples, and control samples (gauzes without microspheres) in distilled water ( $\mathrm{pH}$ 5.5). The distilled water was used to mimic skin pH (5.5-5.6) [20]. At determined time points, aliquots were taken and piroxicam release was monitored by absorbance measurements at 353nm using a He $\lambda$ ios $\gamma$ ThermoSpectronic spectrophotometer (Unicam). The quantification of piroxicam released was established by a piroxicam standard absorbance curve. The release studies were carried out in triplicate and for a period of 6 days. Results are reported as mean value $\pm \mathrm{SD}$.

\subsection{Evaluation of the anti-inflammatory effect of microspheres applied to human skin fibroblasts}

Normal human skin fibroblasts (cell line BJ-5ta) were purchased from the American type culture collection (ATCC). The cells were maintained according to ATCC recommendations (4 parts of Dulbecco's modified Eagle's medium containing $4 \mathrm{mM}$ L-glutamine, $4.5 \mathrm{~g} \mathrm{~L}^{-1}$ glucose, $1.5 \mathrm{~g} \mathrm{~L}^{-1}$ sodium bicarbonate and 1 part of Medium 199, supplemented with $10 \%(\mathrm{v} / \mathrm{v})$ of fetal bovine serum, $1 \%(\mathrm{v} / \mathrm{v})$ penicillin/streptomycin solution and $10 \mu \mathrm{g} \mathrm{mL}^{-1}$ Hygromycin B). The cells were maintained at $37^{\circ} \mathrm{C}$ in a humidified atmosphere of $5 \% \mathrm{CO}_{2}$. Culture medium was refreshed every two to three days. Cells were seeded at a density of $10 \times 10^{3} \mathrm{cells} / 1000 \mu \mathrm{L} /$ well on 12 -well tissue culture polystyrene (TCPS) plates (TPP, Switzerland). One day after seeding, the cells were exposed over $24 \mathrm{~h}$ to dif- ferent microsphere concentrations added to fresh culture medium as well as to different control solutions (BSA solution; piroxicam solution).

After incubation, the cells were washed in PBS (1x) and RNA was isolated from cell cultures, according to the SV Total RNA isolation system protocol from Promega. The quality and concentration of RNA samples were evaluated using a Nanodrop Spectrophotometer before conversion to cDNA using the high-capacity cDNA reverse transcription kit (BIO-RAD) with thermal cycling conditions running for $5 \mathrm{~min}$ at $25^{\circ} \mathrm{C}, 30 \mathrm{~min}$ at $42^{\circ} \mathrm{C}$ and $5 \mathrm{~min}$ at $85^{\circ} \mathrm{C}$. The anti-inflammatory effect of the proteinaceous microspheres was evaluated as the ability to downregulate the gene expression of COX-2 in human skin fibroblasts by real-time PCR. Taqman Universal Master Mix II and the predesigned/preformulated primer and probe sets, TaqMan Gene Expression Assays, were purchased from Applied Biosystems (California, USA). The $\Delta \Delta \mathrm{Ct}$ method was applied using mitochondrial ATPase6 as thehousekeeping gene. PCR amplification was performed according to the manufacturer's instructions on a CFX96 Touch Real-Time PCR Detection System (Bio-Rad Laboratories Hercules, CA 94547).

\subsection{Wound-healing assay}

Tissues were cultured at the air-liquid interface in tissue culture inserts placed in six-well plates. Upon receipt, the tissues were placed into new six-well plates containing $2.5 \mathrm{~mL}$ of fresh culture medium and kept at $37^{\circ} \mathrm{C}, 5 \% \mathrm{CO}_{2}$ overnight. Burn wounds were obtained by placing a cautery on top of the tissue for $10 \mathrm{~s}$. The gauze samples were place on top of the tissue covering the wounded area. Two burn wounds per tissue were done to control the wound size and healing was evaluated in two independent assays. Skin equivalents without functionalised gauzes and treated with a commercial collagen dressing, Suprasorb C (Lohmann \& Rauscher, Germany), were used as controls.

Healing was evaluated over a period of six days and the tissues were then evaluated histologically. Skin equivalents were fixed in $4 \%$ formaldehyde solution at room temperature. Afterwards, paraffin-embedded tissue sections $4 \mu \mathrm{m}$ thick were obtained and stained with haematoxylin and eosin by a standard protocol. All sections were observed under a light microscope (Olympus IX71).

\section{Results and discussion}

\subsection{Attachment of proteinaceous microspheres, containing piroxicam, to cotton and non-woven gauzes}

Considering the special advantages and high potential for the application of microstructured materials in the textile 
industry, especially for producing high-performance biomedical textiles, this study reports the application of microstructured proteinaceous material containing an anti-inflammatory drug for biomedical applications.Two different methods, silent (incubation) and sonochemical treatments, were used to attach BSA microspheres, containing the anti-inflammatory drug, to cotton and nonwoven gauzes.

For the silent methodology, the microspheres were previously prepared and characterised.

In our previous work, matrices based on BSA protein were produced and characterised [21]. Briefly, the characterisation results presented earlier indicated that smaller sizes were obtained with 95:5 aqueous/organic phase and $8 \%$ stabiliser. The proteinaceous microspheres obtained were spherical, homogeneous particles with smooth surfaces [21]. The efficiency of microsphere formation was determined with the Lowry assay [22],resulting in about $100 \%$ yield. Furthermore, the results showed high entrapment efficiency $(\approx 80 \%)$ of piroxicam in BSA microspheres. These proteinaceous microspheres, containing piroxicam, had a $Z$-average value of $(300 \pm 20) \mathrm{nm}$ with a particle size distribution, determined as a polydispersity index $(\mathrm{PDl})$, of $0.045 \pm 0.001$. The previous results also demonstrated that these particles had a zeta-potential of around $(-5 \pm 0.9) \mathrm{mV}$, indicating the presence of negative charges distributed on the surface of the microspheres. Moreover, the stability results proved that BSA microspheres were stable for at least two months, since the Z-average and the zeta-potential measurements gave almost the same values. Therefore, these conditions were used as standard conditions for this study.

After this extensive characterisation, the protein microspheres were incubated with gauzes (cationised and non-cationised) in a shaking water bath, at $25^{\circ} \mathrm{C}$ and $50 \mathrm{rpm}$

In another set of experiments, the attachment of microspheres was carried out in a one-step reaction using the ultrasound system. This attachment procedure did not lead to significantly different physico-chemical characteristics of BSA microspheres containing piroxicam. The efficiency of microsphere formation was about 100\% and the entrapment efficiency of piroxicam was around $76 \%$. The higher entrapment efficiency can be explained by their sparing solubility in water due to drug-albumin interactions. Indeed, piroxicam revealed a higher partition capacity in liposomes and consequently present a higher $K_{\mathrm{p}}$ value [23]. Additionally, location studies in the lipidic layer showed that piroxicam could be positioned with the hydrophilic part oriented toward the polar part of the membrane and the hydrophobic segment in the upper part of the lipophilic tails [23]. Thus, piroxicam must be aligned between the albumin molecules in the protein layer and inside the microspheres. Piroxicam entrapment efficiency is an important factor because the release rate is usually dependent on the drug concentration gradient
[24]. Furthermore, higher levels of drug incorporation lead to a wider concentration gap between the polymeric microspheres and the release medium and can cause a higher diffusion rate.The values of zeta-potential were also very similar $((-5 \pm 0.5) \mathrm{mV})$, as well as the $Z$-average and $\mathrm{PDl}$ values $((280 \pm 10) \mathrm{nm}$ and $0.065 \pm 0.001$, respectively $)$.

Taking into account that microspheres have a negative surface charge, our aim was the activation of the surface of the gauzes to obtain a positive charge. Consequently, greater electrostatic interactions between the negative surface charge of BSA microspheres and the positive surface charge of the gauzes was expected.

The addition of cationising products to cotton is used extensively to improve anionic dye fastness [25]. This study reports not only the cationisation of cotton gauzes, but also of the non-woven gauzes using PDDA. The quaternary ammonium group, ${ }^{+} \mathrm{N}\left(\mathrm{CH}_{3}\right)_{3}$, has a very high positive charge and can thereby lead to the formation of ionic bonds (salt linkages) with negatively charged anionic groups, such as those found in a wide array of anionic dye classes or carboxyl-containing compounds [26]. The reason to submit the non-woven gauze to cationisation is due to the presence of viscose (67\%), containing hydroxyl groups,in the cotton fibre. These hydroxyl groups acquire a negative charge when they are ionisable, decreasing the electrostatic interactions with the BSA microspheres and thus the need for cationisation of the gauzes.

The cationised and control samples (cotton and nonwoven gauzes not cationised) were dyed with Comassie blue, a known acid dye, to evaluate the cationisation efficiency. When dyed, ionic bonding with fibre cationic sites accounts for the fixation of coloured anions in the dyed material. The efficiency of the cationisation process was determined based on colour strength $(K / S)$ (Table 1). Comparing the $K / S$ values of the cationised and the noncationised gauzes (cotton and non-woven), it is possible to conclude that Comassie dye has greater affinity for the cationised fibre than that of the non-cationised fibre, demonstrating the strong electrostatic interactions between the negative charge of dye molecules and the positive charge present at the gauze surfaces.

Table 1. $K / S$ values $(\lambda=600 \mathrm{~nm})$ for the cationised samples (cotton and non-woven gauzes represented as $\mathrm{CO}$ cat and NW cat, respectively) and the control samples (non-cationised cotton and non-woven gauzes, represented as CO control and NW control, respectively) dyed with Comassie brilliant blue G250. K/S was calculated according to Eq. (3). Values reported are the mean \pm SD of at least three different batches of each microsphere formulation attached to gauze bandages

\begin{tabular}{lc}
\hline Samples & $K / S$ \\
\hline CO (control) & $1.51 \pm 0.3$ \\
CO cat & $23.8 \pm 0.9$ \\
NW (control) & $1.8 \pm 0.5$ \\
NW cat & $25.0 \pm 0.7$ \\
\hline
\end{tabular}


The proposed methods to attach piroxicam-loaded BSA microspheres to cotton and non-woven gauze bandages (cationised and non-cationised) were evaluated using ninhydrin. After 30 min of reaction, a colour change in the gauze bandages from light yellow to purple was observed, indicating reaction between the protein microspheres and ninhydrin. It is known that ninhydrin reacts with amino acids or proteins to generate a visible purple colour in the presence of amines [18].

Figure 1 depicts the development of a blue colour, in all samples (cationised and non-cationised), confirming the presence of protein at the surface of the gauzes. These results suggest, however, a more pronounced blue colour for the cationised samples, evidencing greater protein attachment to the positively charged surfaces. Furthermore, ultrasound treatment seems to promote more uniform protein transfer from solution to the gauze surface, which translates to more uniform colour development.

\subsection{Morphology of gauze bandages with microspheres}

Morphological analysis of the fibres was carried out by SEM, which enabled qualitative 3D imaging of surface features. It is clearly illustrated that, with either methodologies, it was possible to attach BSA microspheres, containing piroxicam, to the cotton and non-woven gauzes (Fig. 2).The images also suggest that the presence of positive charges on the surfaces of the gauzes result in microspheres attachment because it is possible to visualise a layer of microspheres almost completely covering the surface of the gauze fibres. These data corroborate the ninhydrin results.

\subsection{In vitro release profile of piroxicam from cotton and non-woven gauze bandages}

The release behaviour of polymeric microspheres is one of the most important features of drug/polymer formulations for sustained drug delivery. The release profile of BSAbased particles was thus studied (Fig. 3) for wet gauze bandages (immediately after ultrasound or incubation treatment) and dried gauze bandages (samples dried at room temperature).

The release profiles shown have two distinct patterns: an accentuated initial burst of release during the first $6 \mathrm{~h}$ followed by a stagnant phase of release for the remaining time. The burst of piroxicam is associated with piroxicam molecules dispersing close to the surface of the microspheres, which diffuse outwards at the beginning of incubation (Fig. 3). The release of a compound from a matrix is governed by several factors, such as the nature and size of the particles and also the nature of the compound being entrapped. From the SEM results discussed previously, it was concluded that ultrasound produced a higher

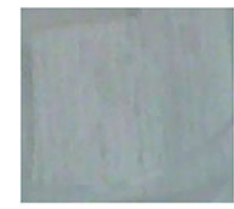

NW Control

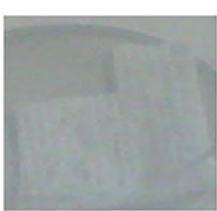

NW cat Control

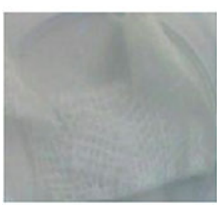

CO Control

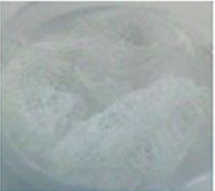

CO cat Control

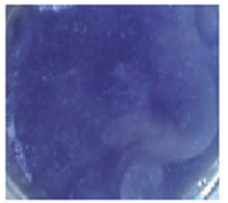

NW Incubation

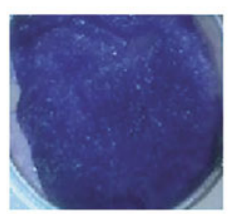

NW cat Incubation

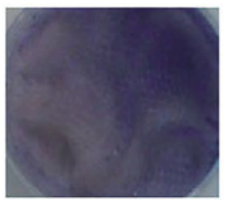

CO Incubation

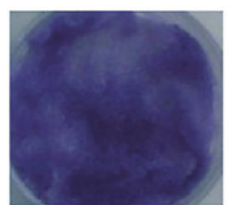

CO cat Incubation

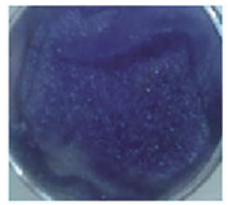

NW Ultrasound

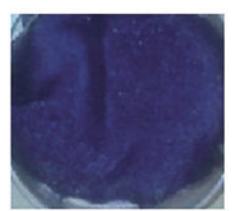

NW cat Ultrasound

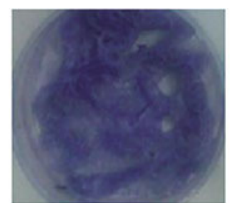

CO Ultrasound

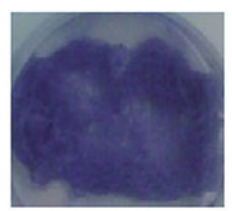

CO cat Ultrasound
Figure 1. Images of colour developed after 30 min of ninhydrin application on the microspheres attached to cotton and non-woven gauze bandages by sonication or incubation methods (cotton and non-woven gauzes represented as $\mathrm{CO}$ and NW, and cotton and non-woven gauzes cationised represented as $\mathrm{CO}$ cat and NW cat, respectively). The control samples were incubated or sonicated with PBS (non-cationised gauzes: cotton and non-woven without microspheres, denoted CO control and NW control, respectively; cationised gauzes: cotton and non-woven without microspheres, denoted CO cat control and NW cat control, respectively).

content of proteinaceous microspheres. The results show that the BSA particles prepared by ultrasound released more piroxicam (Fig. 3); this can be attributed to the fact that, in liquid media, ultrasound enhances mass transport of constituents (protein microspheres) in a non-homogeneous fashion, allowing efficient attachment to the gauze fibres. The beneficial use of ultrasound is realised through its chemical, mechanical or physical effects on the process or product [27]. Conversely, attachment by incubation with constant stirring is not sufficient to promote efficient treatment, which can explain the lower release of piroxicam. The amount of piroxicam released can be correlated with the quantity entrapped in microspheres effectively attached to the surface of the fibres.

Incubation and ultrasound treatment with the characteristics described appear to yield the same release profilesforpiroxicam from cationised gauze bandages (Fig. 3). 

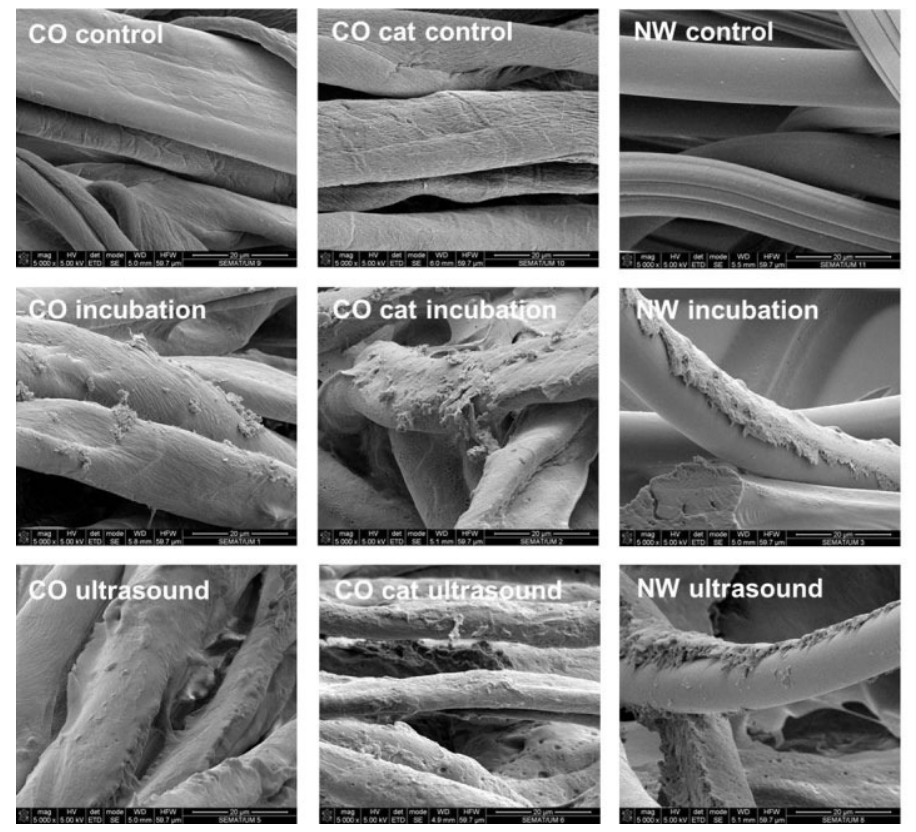
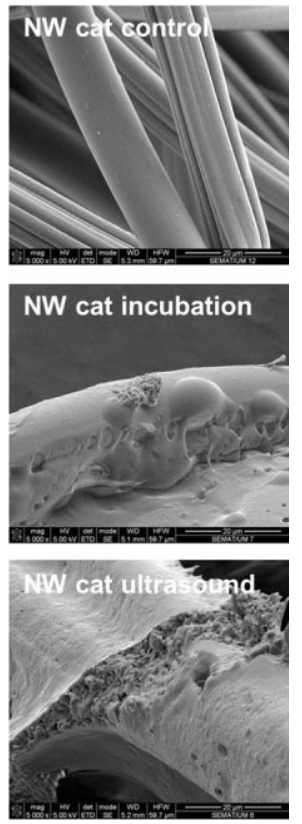

Figure 2. SEM images (magnification of $5000 x$ ) of cotton gauzes and non-woven bandages.
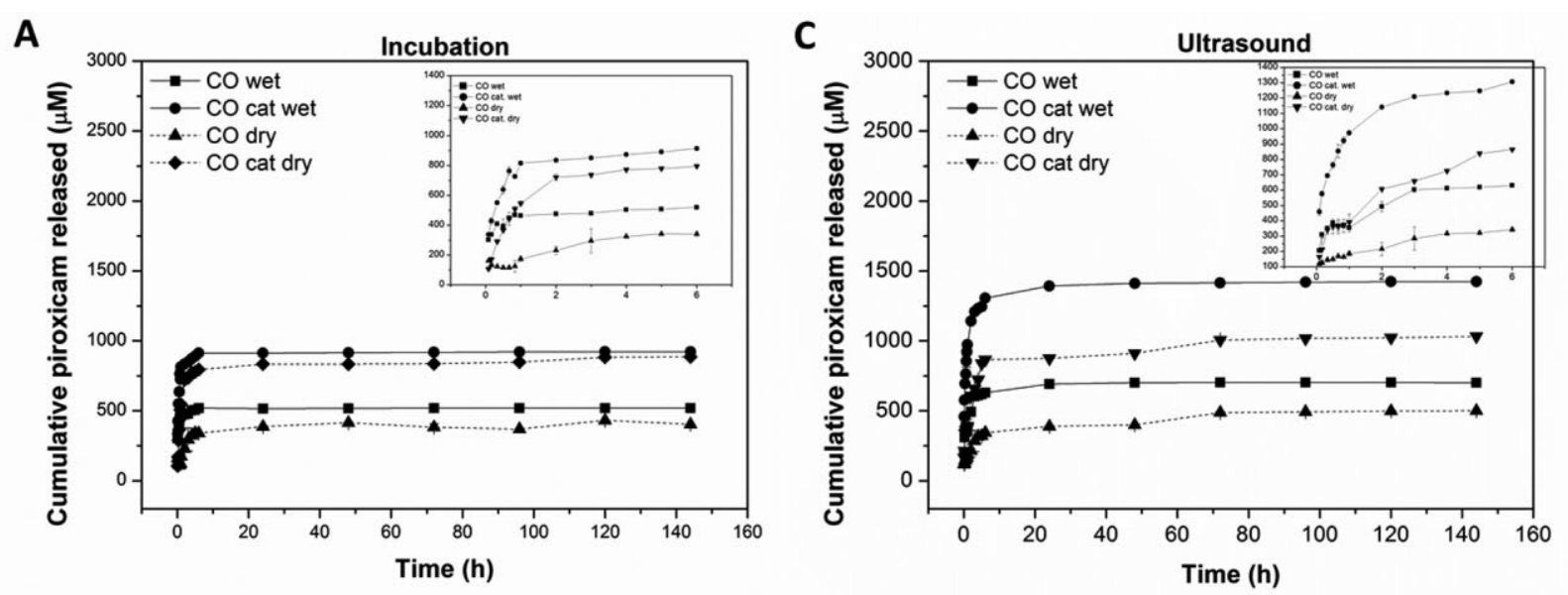

B

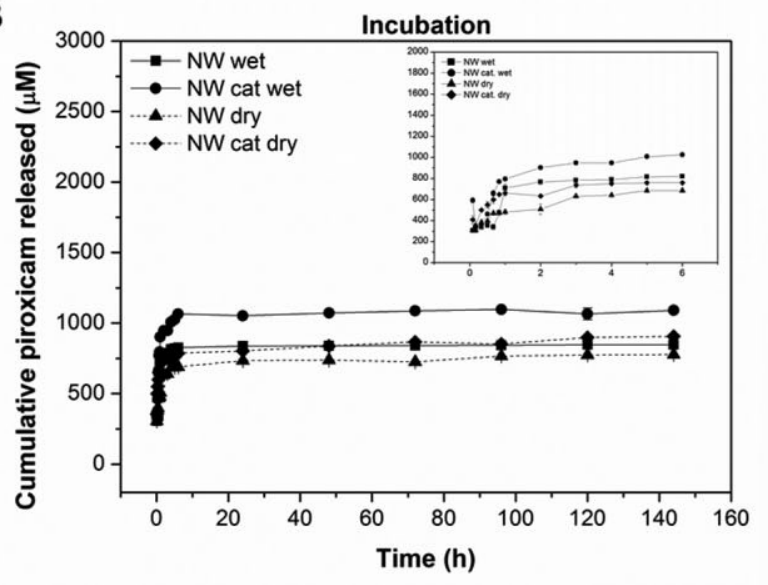

D

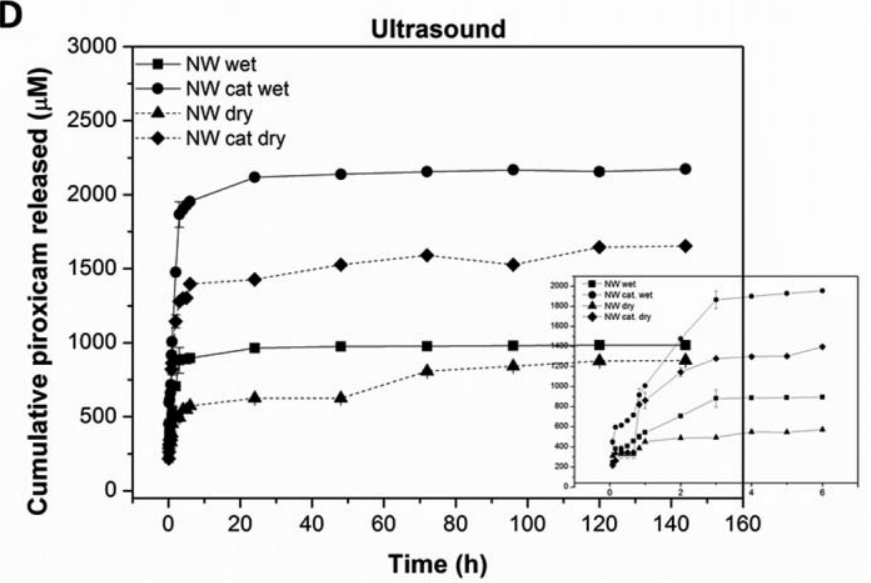

Figure 3. In vitro release profiles of piroxicam entrapped in BSA microspheres from cotton and non-woven gauzes bandages: incubation treatment (A, B) sonochemical treatment $(C, D)$. Each point represents the mean \pm SD values from three different batches. The inset values show the release profile over the first 6 h. 
As stated, the cationisation process promotes the presence of positive charges on the gauze surface that favour the attachment of BSA microspheres, which have a negative surface charge, to the structure of the gauze material (cotton and non-woven). Therefore, the promotion of electrostatic interactions between the microspheres and the cationised gauzes can lead to increased release of piroxicam. These data also indicate the lowest concentration of piroxicam released from dry gauze bandages. This result can be attributed to the fact that the drying process can induce premature disruption of BSA microspheres and, consequently, a lost in piroxicam content. It is also necessary to note that, in dried samples, hydration of the textile material may be more difficult or slower and, consequently, the diffusion of piroxicam to the medium may be decreased.

Interestingly, gauze porosity has a significant influence on BSA microsphere attachment, affecting the drugdelivery concentrations. The results show that,independent of the methodology used to immobilise the BSA microspheres, the non-woven gauze releases more piroxicam over time. In this way, being more closed and compact than cotton gauzes, this type of fibre structure can offer better support for the attachment of microspheres.

\subsection{Anti-inflammatory properties and wound healing}

The biocompatibility of piroxicam entrapped in proteinaceous devices was previously tested [21] and identified the range of concentrations (75-300 $\mathrm{mg} \mathrm{L}^{-1}$ ) as non-cytotoxic for two cell types, including BJ-5ta.

In this study, the anti-inflammatory potential of these particles, containing piroxicam, was confirmed. It is well known that the action of NSAIDs is mediated by its capacity to inhibit cyclo-oxygenase (COX) activity, in particular, an inducible isoform (COX-2), found in areas of inflammation and in the brain [28]. It has been suggested that the anti-inflammatory actions of NSAIDs are due to the inhibition of COX-2, whereas unwanted side effects are due to the inhibition of COX-1 [29]. For this reason, expression of the cox-2 gene was examined in human skin fibroblasts (BJ-5ta) in the presence of microspheres, containing or lacking piroxicam.

Figure 4 shows relative cox- 2 gene expression in the presence of proteinaceous microspheres containing piroxicam $(\mathrm{PM}+\mathrm{Px})$ at two different concentrations, microspheres without piroxicam (PM) and a control solution of soluble BSA and different concentrations of piroxicam. All piroxicam solutions decreased the levels of relativecox-2 gene expression as expected;the highest concentration of piroxicam ( $180 \mu \mathrm{M}$ equivalent to the maximum amount encapsulated) is the most effective. The physical process of microsphere formation has some influence on the anti-inflammatory capacity of the isolated compo-

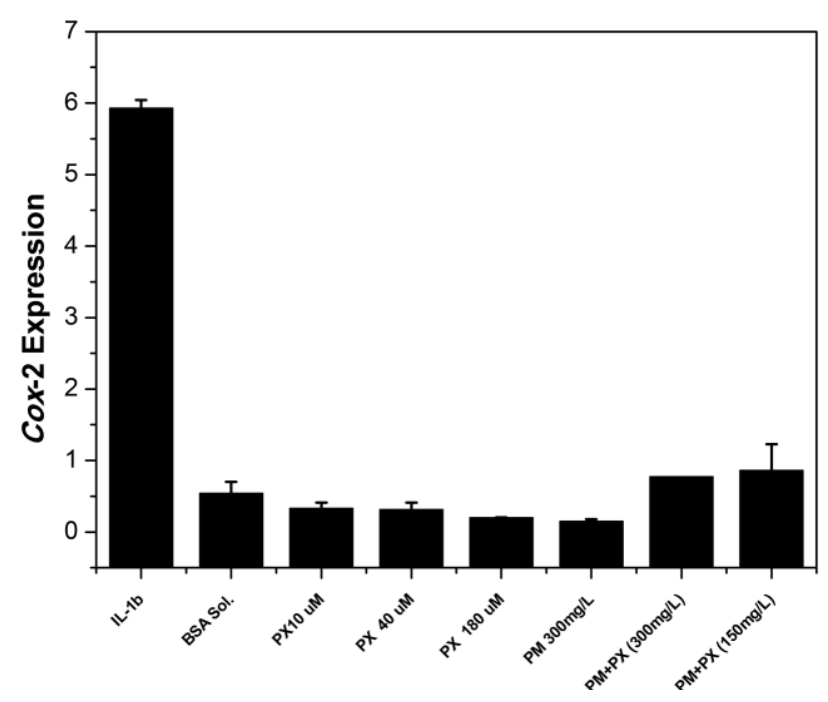

Figure 4. Relative cox-2 gene expression, measured by real-time PCR, in the human fibroblast cell line BJ5ta in the presence of different concentrations proteinaceous microspheres, containing piroxicam, $(\mathrm{PM}+\mathrm{Px}$ $300 \mathrm{mg} \mathrm{L}^{-1}$; $\left.\mathrm{PM}+\mathrm{Px} 150 \mathrm{mg} \mathrm{L}^{-1}\right)$; protein microspheres without piroxicam (PM $300 \mathrm{mg} \mathrm{L}^{-1}$ ); BSA solution; different piroxicam concentrations (10; 40 and $180 \mu \mathrm{M})$ and in the presence of interleukin 1-beta (IL-1 $\beta$ ). Results show the mean values of two replicates from two experiments carried out independently.

nents. PMs with piroxicam have less anti-inflammatory effect than the isolated components at similar concentrations; the opposite is true for PMs without piroxicam. The data demonstrates for this cell model that these materials have the ability to reduce the inflammation process mediated by COX-2 in human fibroblasts to levels similar to those found in the control experiment.

The biocompatibility of any material should also be assessed by exposing THP-1 human macrophages to it and evaluating the production and release of the pro-inflammatory cytokine TNF- $\alpha$. Preliminary results suggested that functionalised gauze bandages induced the production of low levels of TNF- $\alpha$ protein after $48 \mathrm{~h}$ of incubation (data not shown).

To determine if the different materials affected the kinetics of wound healing differently, the materials were applied, wet, on top of the skin equivalents immediately after the burn wounds were inflicted and histological analysis was performed six days later.

Optimisation of the attachment of proteinaceous particles to gauze bandages was achieved with ultrasound. Therefore, functionalised samples prepared with ultrasound were analysed for their capacity to promote wound healing.

Epidermal regeneration of a wound is a complex process in which residual epithelial cells proliferate in an integrated manner to form an intact epidermis. In burns, a number of epithelial cells survive in hair follicles and 

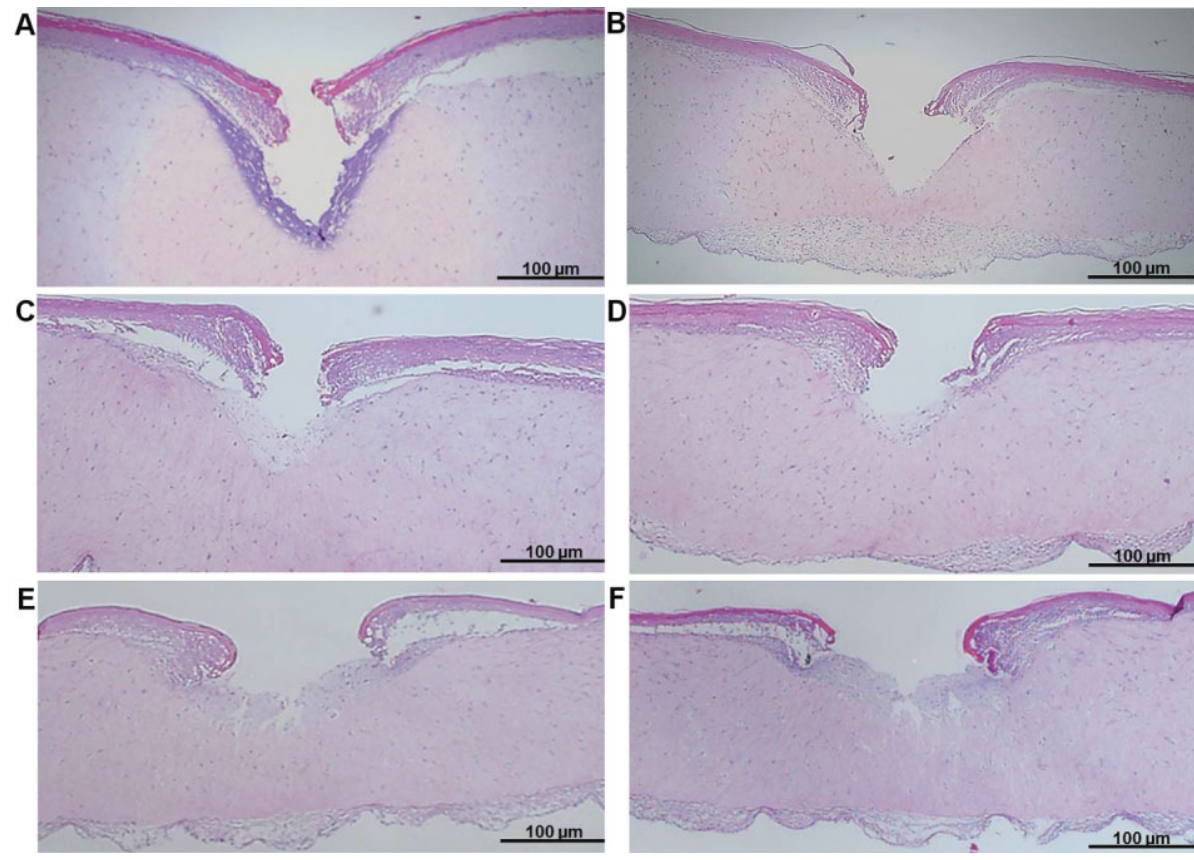

Figure 5. Histological cross-sections of wound burnsstained with haematoxylin/ eosin, in which $(\mathbf{A})$ is the wound immediately after the burn, $t=0$ (control); (B) is the wound after six days without any treatment (control); (C) is the wound with Suprasorb C after six days (control); (D) is the wound with microspheres after six days; (E) is the wound with microspheres on NW cat after six days, and $(\mathbf{F})$ is the wound with microspheres on $\mathrm{CO}$ cat after six days. sweat glands. Prevention of infection is the primary aim of the treatment of burn wounds to allow for the optimal regeneration of lost cells. Thus, a moist environment is best for epithelialisation and wound healing [30].

Figure 5 shows that it is possible to observe the architecture and relative size in histological sections of the wounds six days post-wounding. The results revealed that the proteinaceous microspheres, containing piroxicam and applied to cotton and non-woven gauzes, induced cell proliferation and migration to the wound site, especially for wounds treated with cationised gauzes. The results obtained are comparable with those for the commercial collagen dressing; Suprasorb C, used for several types of wounds, including burn wounds.

Macroscopic observations of the wounds indicate that the control samples (Fig. 5A) are characterised by the lack of epithelium and the dermis is covered with a crust from burning. Six days later, the crust had disappeared from the control sample (Fig. 5B) and samples exposed to different materials showed healing (Fig. 5C-F).

Moreover, wounds in contact with gauzes induced cell proliferation and migration (most probably keratinocytes and fibroblasts) from the margins to the wound core, which would result in faster re-epithelisation and wound closure.

During the healing of an acute wound, re-epithelisation occurs from the margin to its core. Therefore, the results indicated that wound size reduction was greater in the cationised non-woven gauze sample even when compared with the commercial control (Suprasorb C). Histological examination was done with the section obtained in the centre of wound. Thus, the results reflect the healing improvement promoted by contact with the BSA mi- crospheres, containing piroxicam, attached to the gauzes.

From the histological results obtained, it can also be noted that wounds healed in contact with BSA microspheres, attached or not to cationised gauzes, were almost completely closed and covered with new epithelium, which was not the case for the controls.

The characterisation of the non-woven gauzes obtained by cationised treatment suggested that these offer greater availability of microspheres for the wound-healing process.

It has been proposed that NSAIDs can result in a modest inhibition of the initial inflammatory response and its symptoms [31]. Recent studies also demonstrated that the use of vegetable oils in protein formulations had the ability to reduce the activity of elastase [13]. Nevertheless, the inclusion of both NSAID and vegetable oil in the microsphere's composition and attachment to gauzes was exploited for the first time, allowing us to develop a novel wound dressing for biomedical applications.

\section{Concluding remarks}

This study demonstrates the capacity of proteinaceous microspheres, containing an anti-inflammatory drug, to become attached to different gauzes, cotton and non-woven. The continuous application of shear stress within the bulk solution from the mixing effect provided by stirring or ultrasound waves was able to promote the attachment of BSA particles to gauze bandages. Furthermore, the chemical activation of gauzes, using PDDA as a cationising reagent, improved the content of BSA microspheres 
at the surfaces of the fibres and, consequently, a higher concentration of piroxicam was released. However, most drug was released when the attachment process was performed by the sonochemical method. The short treatment time (3 min) improves the mass transport effects by enhancing attachment of the microspheres. The promotion of attachment of the microspheres to gauzes using ultrasound was achieved by a one-step reaction, signifying a reduction in product consumption, shorter process time and a greater uniformity of treatment.

The release of piroxicam entrapped in BSA microspheres was dependent on the structure of the gauze. The fastest and highest concentration of piroxicam released was reached when the proteinaceous microspheres were attached to non-woven gauzes. Moreover, dermal burn healing experiments using human skin equivalents showed that the application of our material accelerated re-epithelisation and wound closure.

The results achieved indicate that these functionalised gauze bandages may potentially be used as powerful bioactive carriers for wound healing. Furthermore, such drug carriers can be helpful for several pathologies where the bioactivity of piroxicam is expected to be therapeutically relevant.

We would like to acknowledge financial support from the European project Lidwine (contract no. NMP2-CT-2006026741), and POPH/FSE for Co-financing and Fundação para a Ciência e a Tecnologia (FCT) for fellowship SFRH/BPD/38939/2007 awarded to Helena Ferreira. The work at CBMA was supported by FEDER through POFC-COMPETE and by national funds from FCT through the project PEst-C/BIA/UI4050/2011.

The authors declare no conflict of interest.

\section{References}

[1] Boateng, J. S., Matthews, K. H., Stevens, H. N. E., Eccleston, G. M., Wound healing dressings and drug delivery systems: A review. J. Pharm. Sci. 2008, 97, 2892-2923.

[2] Wollina, U., Heide, M., Muller-Litz, W., Obenauf, D., Ash, J., Functional textiles in prevention of chronic wounds, wound healing and tissue engineering, in Elsner, P., Hatch, K., Wigger-Alberti, W. (Eds.), Textiles and the skin. Curr. Probl. Dermatol. Karger, Basel, 2003, 31, 82-97.

[3] Harding, K., Cutting, K., Price, P., The cost-effectiveness of wound management protocols of care. Br. J. Nurs. 2000, 10, 216-217.

[4] Jones, V. J., The use of gauze: Will it ever change? Int. Wound J. 2006, 3, 79-88.

[5] Degim, Z., Use of microparticulate systems to accelerate skin wound healing. J. Drug Target. 2008, 16, 437-448.

[6] Dahl, J. B., Kehlet, H., Non-steroidal anti-inflammatory drugs: Rationale for use in several postoperative pain. Br. J. Anaesth. 1991, 66, 703-712.

[7] Elsayed, M. M. A., Abdallah, O. Y., Naggar, V. F., Khalafallah, N. M., Lipid vesicles for skin delivery of drugs: Reviewing three decades of research. Int. J. Pharm. 2007, 332, 1-16.
[8] Wang, B., Siahaan, T., Soltero, R. (Eds.), Drug Delivery: Principles and Applications, Wiley, New York, 2005.

[9] Antonio, F., Guillem, R., Sonia, T., Clara, M.et al., Cross-linked collagen sponges loaded with plant polyphenols with inhibitory activity towards chronic wound enzymes. Biotechnol. J. 2011, 6, 1208-1218.

[10] Vasconcelos, A., Gomes, A. C., Cavaco-Paulo, A., Novel silk fibroin/elastin wound dressings. Acta Biomater. 2012, 8, 3049-3060.

[11] Couvreur, P., Gref, R., Andrieux, K., Malvy, C., Nanotechnologies for drug delivery: Application to cancer and autoimmune diseases. Prog. Solid State Chem. 2006, 34, 231-235.

[12] Wang, X., Wenk, E., Matsumoto, A., Meinel, L.et al., Silk microspheres for encapsulation and controlled release. J. Controlled Release 2007, 117, 360-370.

[13] Silva, R., Ferreira, H., Vasconcelos, A., Gomes, A., Cavaco-Paulo, A., Sonochemical proteinaceous devices for wound healing. Adv. Exp. Med. Biol. 2012, 733, 155-164

[14] Edwards, P. D., Bernstein, P. R., Synthetic inhibitors of elastase. Med. Res. Rev. 1994, 14, 127-194.

[15] Cassano, R., Trombino, S., Ferrarelli, T., Barone, E.et al., Synthesis, characterization, and anti-inflammatory activity of diclofenacbound cotton fibers. Biomacromolecules 2010, 11, 1716-1720.

[16] Suslick, K. S., Grinstaff, M. W., Protein microencapsulation of nonaqueous liquids. J. Am. Chem. Soc. 1990, 7807-7809.

[17] Subramanian Senthil Kannan M. , Gobalakrishnam M., Kumaravel S., Nithyanadan R.et al., Influence of cationization of cotton on reactive dyeing. Journal of Textile and Apparel, Technology and Management 2006, 5, 1-22.

[18] Harold, R. W., Textiles: Appearance analysis and shade sorting. Textile Chemical Coloration 1987, 19, 23-31.

[19] Macdonald, R., Colour Physics for Industry, Society of Dyers and Colourists, Bradford, 1987

[20] Braun-Falco, O., Korting, H., Normal pH value of human skin. Hautarzt 1986, 37, 126-129

[21] Silva, R., Ferreira, H., Carvalho, A. C., Gomes, A. C., Cavaco-Paulo, A., Protein microspheres as suitable devices for piroxicam release. Colloids Surf., B 2012, 92, 277-285.

[22] Lowry, O. H., Rosebrough, N. J., A. Lewis Farr, Randall, R. J., Protein measurement with folin-phenol reagent. J. Biol. Chem. 1951, 193, 265-275.

[23] Lúcio, M., Ferreira, H., Lima, J. L. F. C., Reis, S., Interactions between oxicams and membrane bilayers: An explanation for their different COX selectivity. Med. Chem. 2006, 2, 447-456.

[24] Xu, Y., Du, Y., Effect of molecular structure of chitosan on protein delivery properties of chitosan nanoparticles. Int. J. Pharm. 2003, 250, 215-226.

[25] Seong, H. S., Ko, S. W., Synthesis, application and evaluation of cationising agents for cellulosic fibres. J. Soc. Dyers Colour. 1998, 114, 124-129.

[26] Hashem, M., El-Bisi, M., Sharaf, S., Refaie, R., Pre-cationization of cotton fabrics: An effective alternative tool for activation of hydrogen peroxide bleaching process. Carbohydr. Polym. 2010, 79, 533-540.

[27] Jambrak, A. R., Mason, T. J., Lelas, V., Herceg, Z., Herceg, I. L., Effect of ultrasound treatment on solubility and foaming properties of whey protein suspensions. J. Food Eng. 2008, 86, 281-287.

[28] Fröhlich, J. C., Prostaglandin endoperoxide synthetase isoenzymes: The clinical relevance of selective inhibition. Ann. Rheum. Dis. 1995, 54, 942-943.

[29] Brooks, P., Use and benefits of nonsteroidal anti-inflammatory drugs. Am. J. Med.1998, 104, 9S-13S.

[30] Subrahmanyam, M., A prospective randomised clinical and histological study of superficial burn wound healing with honey and silver sulfadiazine. Burns 1998, 24, 157-161.

[31] Almekinders, L. C., Anti-inflammatory treatment of muscular injuries in sport: An update of recent studies. Sports Medicine 1999, 28, 383-388. 